\title{
Partial protection against genital reinfection by immunization of guinea-pigs with isolated outer-membrane proteins of the chlamydial agent of guinea-pig inclusion conjunctivitis
}

\author{
Byron E. Batteiger, ${ }^{1 *}$ Roger G. Rank, ${ }^{2}$ Patrik M. Bavoil ${ }^{3}$ and Lee S. F. Soderberg ${ }^{2}$ \\ ${ }^{1}$ Department of Medicine, Indiana University School of Medicine, Indianapolis IN 46202-5124, USA \\ ${ }^{2}$ Department of Microbiology and Immunology, University of Arkansas for Medical Sciences, Little Rock, \\ Arkansas 72205, USA \\ ${ }^{3}$ Department of Microbiology and Immunology, University of Rochester Medical Center, Rochester, NY 14625, USA
}

(Received 18 January 1993; revised 18 June 1993; accepted 2 August 1993)

\begin{abstract}
Because partial protection against reinfection is induced by experimental infection in the guinea-pig model of genital chlamydial infection, we sought to induce immunity by immunization. Female guinea-pigs were immunized subcutaneously with the major outer-membrane protein (MOMP) and the $61 \mathrm{kDa}$ cysteine-rich outer-membrane protein (61 kDa) of the agent of guinea-pig inclusion conjunctivitis (GPIC) eluted from SDS-polyacrylamide gels (SDS-MOMP, SDS-61 kDa). Post-immunization sera and secretions contained antibodies to the SDS-purified proteins at high titre as measured by immunoblotting, whereas enzyme immunoassays (EIA) using whole elementary bodies as antigen showed significantly lower titres $(P<0.001)$. Likewise, blastogenic responses of peripheral mononuclear cells to GPIC elementary bodies were weak. Animals immunized with SDS-MOMP and SDS-61 kDa were fully susceptible to intravaginal challenge, as were control animals immunized with buffer without protein. Another group of animals were immunized with material prepared by extraction of chlamydial outer-membrane complexes with octyl $\beta$-D-glucopyranoside (OGP) and dithiothreitol, which consisted largely of MOMP (OGP-MOMP). In contrast to the SDS-MOMP group, sera and secretions in the OGP-MOMP group showed high titres in EIA, and high titre antibodies to MOMP by immunoblot; however, most animals also had antibodies to $61 \mathrm{kDa}, 72 \mathrm{kDa}$ and ca. $84 \mathrm{kDa}$ outer-membrane proteins. OGP-MOMP animals were partially protected against genital challenge as evidenced by low inclusion scores compared to control animals, although duration of infection measured by culture isolation was similar to controls. Immunoblot analysis of sera from immunized animals and from a group of immune animals post-infection was performed using recombinant fusion peptides containing the four variable domains of MOMP. No consistent differences in reaction patterns were observed when sera from protected and non-protected animals were compared. Thus, a highly refined outermembrane preparation is capable of producing partial immunity to genital infection. Further study is required to determine whether the protection is due to MOMP itself or to other outer-membrane proteins found in small amounts in the OGP-MOMP immunogen. The results suggest the possibility that discontinuous MOMP epitopes could play a role in inducing a protective immune response in the guinea-pig model, a concept that requires further evaluation.
\end{abstract}

\section{Introduction}

Development of a safe effective chlamydial vaccine has been considered an important goal because of the high

*Author for correspondence. Tel. +1 3172748115 ; fax +1 317274 1587.

Abbreviations: EB, elementary body; EIA, enzyme immunoassays; GPIC, guinea-pig inclusion conjunctivitis; MOMP, major outermembrane protein; OGP, octyl $\beta$-D-glucopyranoside; VD, variable domain. prevalence and considerable morbidity associated with both trachoma and genital infections due to Chlamydia trachomatis. One difficulty in vaccine development is that strains of $C$. trachomatis, excepting the mouse pneumonitis (MoPn) strain, are strictly human pathogens. However, several animal models, including conjunctivitis models in primates (Taylor et al., 1988), have proved useful in previous $C$. trachomatis immunization studies. The guinea-pig models of eye and genital infection caused by the naturally-occurring pathogen guinea-pig inclusion conjunctivitis agent (GPIC), a C. psittaci strain, 
have been well-characterized (Batteiger \& Rank, 1987; Rank \& Batteiger, 1989; Rank et al., 1988; Rank \& Barron, 1987). The guinea-pig: GPIC models offer alternative and more accessible experimental systems in which to develop vaccine strategies. We know that a brief period of absolute protection against reinfection results from genital infection in this model, followed by a prolonged period of partial protection (Rank et al., 1988). It is thus reasonable to determine if such protection can be induced artificially.

Another difficulty in vaccine development was noted in trachoma vaccine trials conducted in the $1960 \mathrm{~s}$ (summarized in Schachter \& Dawson, 1978) using inactivated whole elementary bodies. Some immunized patients experienced enhanced eye disease (hypersensitivity) as compared to unvaccinated controls. A GPIC genus-specific hypersensitivity protein capable of inducing such responses in the guinea-pig eye has been described (Morrison et al., 1989). Thus, a subunit vaccine, which would separate protective from deleterious components, would be desirable (Schachter, 1985).

The chlamydial major outer-membrane protein (MOMP) has been most intensively studied as a potential immunogen. A cross-species analysis of available MOMP sequences has revealed a highly conserved primary structure with the exception of four variable domains (VD1-4) (Stephens et al., 1988; Yuan et al., 1989; Zhang et al., 1989). Based on immunoblot analyses of sera from guinea-pigs with GPIC genital infection, VDs 1-4 appear to be immunodominant (B. E. Bavoil \& R. G. Rank, unpublished data). These domains in $C$. trachomatis strains contain serovar-, subspecies- and species-specific determinants (Baehr et al., 1988), some of which are neutralizing in in vitro systems (Baehr et al., 1988; Zhang et al., 1987). In addition, trypsin-sensitive sites in VD2 and VD4 have been implicated in chlamydial attachment (Su et al., 1988). Collectively, these data support the use of MOMP in the development of a subunit vaccine. A vaccine consisting of chlamydial outer-membrane protein complexes protected sheep against systemic infection with the Chlamydia psittaci agent of ovine enzootic abortion (Tan et al., 1990). However, MOMP purified in the presence of SDS did not induce substantial protective immunity when used as an oral immunogen in a monkey conjunctivitis model (Taylor et al., 1988).

The aim of this study was to determine if isolated MOMP, prepared using either SDS or a nonionic detergent and then given parenterally, could induce a protective immune response in the guinea-pig:GPIC model of genital infection. Because antibodies to a cysteine-rich ca. $60 \mathrm{kDa}$ outer-membrane protein are frequently induced in both human (Newhall et al., 1982) and guinea-pig (Batteiger \& Rank, 1987) genital infec- tions, we chose to study the effect of this protein in the model as well.

\section{Methods}

Chlamydiae and protein purification. Elementary bodies (EBs) of the Chlamydia psittaci agent of guinea-pig inclusion conjunctivitis (GPIC) were produced by growth in McCoy cell monolayers and purified using Percoll gradients by established procedures (Batteiger \& Rank, 1987). These EBs were used as starting material to purify proteins, and as antigen in immunoblot, EIA and blastogenesis assays. Chlamydiae for challenge infection were prepared in HeLa cells as previously described (Rank et al., 1988). Chlamydial outer-membrane complexes of GPIC were produced using standard procedures (Batteiger et al., 1985; Caldwell et al., 1981) and used as starting material for production of purified proteins.

The first method used to obtain purified $39 \mathrm{kDa}$ MOMP and the only method employed to obtain the $61 \mathrm{kDa}$ cysteine-rich outer membrane protein was to first resolve outer-membrane proteins on $3 \mathrm{~mm}$ thick SDS-polyacrylamide gels (Caldwell \& Schachter, 1982). The appropriate bands were then excised from the gel, diced into fragments, and eluted into Laemmli electrophoresis buffer (Laemmli, 1970) using an electroelution device (Schleicher and Schuell). Because these proteins were isolated in the presence of SDS, they were designated SDSMOMP and SDS-61 kDa.

Purified MOMP was obtained without the use of SDS by the method of Bavoil et al. (1984) employing octyl $\beta$-D-glucopyranoside (OGP; Boehringer-Mannhein) and dithiothreitol (DTT). Chlamydial outermembrane complexes were incubated in a solution containing $2 \%$ (w/v) Sarkosyl and $40 \mathrm{~mm}-\mathrm{DTT}$ for $1 \mathrm{~h}$ at $37^{\circ} \mathrm{C}$ followed by centrifugation at $100000 \mathrm{~g}$. The supernatant contained most contaminating proteins including the $61 \mathrm{kDa}, 11 \mathrm{kDa}$ and ca. $84 \mathrm{kDa}$ outer-membrane proteins of GPIC (Batteiger \& Rank, 1987). The pellet was then incubated with a solution containing $2 \%(\mathrm{w} / \mathrm{v})$ OGP and $40 \mathrm{~mm}$-DTT for $1 \mathrm{~h}$ at $37^{\circ} \mathrm{C}$ followed by centrifugation at $100000 \mathrm{~g}$. The supernatant contained MOMP as the primary component. These MOMP preparations were designated OGP-MOMP.

The purity of all preparations was assessed using SDSpolyacrylamide gels stained with Coomassie blue R-250. Gels containing OGP-MOMP were scanned using a laser densitometer (LKBBromma), with the proportion of dye binding attributable to MOMP estimated by a programmable integrator (Hewlett-Packard). Protein contents were estimated by the dye-binding assay of Bradford (1976) (Bio-Rad). SDS-MOMP, SDS-61 kDa and OGP-MOMP were used to immunize female guinea-pigs as described below. Two lots of each antigen were prepared independently, and each lot was used to immunize five experimental animals.

Experimental animals, immunizations, and challenge infection. Hartley strain female guinea-pigs, weighing $450-500 \mathrm{~g}$, were obtained from Sasco Laboratories and were housed individually in an environmentally controlled room with a $12 \mathrm{~h}$ light-dark cycle. Hartley strain guinea-pigs are outbred. For each experiment, groups of 10 animals were immunized with either $10 \mu \mathrm{g}$ of a given purified protein preparation $(n=5)$ or mock-immunized with an equal volume of the relevant detergent-containing buffer $(n=5)$. Each animal received three subcutaneous immunizations, each 2 weeks apart. The primary immunization was with Freund's complete adjuvant whereas the second and third immunizations were with Freund's incomplete adjuvant. Each experiment was repeated once, so that a total of 10 animals each were immunized with SDS-MOMP, OGP-MOMP, or SDS-61 kDa, with corresponding mock-immunized control animals.

Guinea-pigs in all groups were challenged 2 weeks following the third immunization with viable HeLa cell-grown chlamydiae administered 
Table 1. Characteristics of plasmid constructs

\begin{tabular}{lclc}
\hline \hline Plasmid & Insert & \multicolumn{1}{c}{ Product } & $\begin{array}{c}\text { Molecular } \\
\text { mass (kDa) }\end{array}$ \\
\hline pATH10 & - & TrpE & 37 \\
pGM101 & Asp141-Ala330 & TrpE-MOMP & 58 \\
pAJC264 & - & LamB & $47 \cdot 8$ \\
pGM1 & Thr67-Arg80 & LamB-VD1 & $49 \cdot 3$ \\
pGM2 & Leu135-Pro147 & LamB-VD2 & $49 \cdot 1$ \\
pGM3 & Thr218-Ser232 & LamB-VD3 & $49 \cdot 2$ \\
pGM4-1 & Pro285-Gly300 & LamB-VD4-1 & $49 \cdot 5$ \\
pGM4-2 & Leu299-Ala312 & LamB-VD4-2 & $49 \cdot 2$ \\
\hline \hline
\end{tabular}

intravaginally. Each animal received approximately $10^{6}-10^{7}$ inclusionforming units contained in $0.05 \mathrm{ml}$ sucrose/phosphate/glutamate buffer, pH $7 \cdot 4$ (Rank et al., 1988). The course of infection was followed by determining the percentage of inclusion-bearing cells on a Giemsastained smear of a scraping from the vaginal vault, and by determining the presence of viable GPIC organisms using cell culture (Rank et al., 1988). Differences in the course of infection were determined using a two-factor (days, treatment group) analysis of variance with repeated measures of one factor (days).

Expression of recombinant MOMP epitopes. Regions of GPIC MOMP which correspond to the four variable domains (VDs) identified in MOMPs from C. trachomatis (Stephens et al., 1988; Zhang et al., 1987) were genetically inserted between serine residues 153 and 154 of the LamB protein of $E$. coli using the expression vector pAJC264 (Charbit et al., 1986). Synthetic oligonucleotides encoding MOMP domains corresponding to VD1 (5'-GATCCGACCGGTAACGCTGCAGCTGACTTTAAAACCGTTGCCGATCGT), VD2 (5'-GATCTGGGTGTTACCGGTACCGACCTGCAGGGTCAGTACCCG), VD3 (5'-GATCCGACTGCAGCTAACTTCCCGCTGCCGCTGACCCCTACCCTCCTAGGT), VD4-1 (N-terminal end) (5'-GATCCTACCGCAATATTAAACCTGACCACCTGGAACCCTACCCTCCTAGGT) and VD4-2 (C-terminal end) (5'-GATCTGGGTGAAGCTACCACCATTAATACCGGCGCCAAATACGCT) were designed to comply with the LamB gene preferred codon usage (Charbit et al., 1986), and to generate two 5-GATC overhangs to allow insertion into the BamHI site of pAJC264. GPIC MOMP VDs were defined by comparison with the reported $C$. trachomatis VD sequences (Yuan et al., 1989; Zhang et al., 1989). More conservative amino acid substitutions were excluded from our design to allow synthesis of single pairs of oligonucleotides each for VD1 and VD2. In the case of VD4, two oligonucleotide pairs were made to generate two distinct hybrids, LamB-VD4-1 and LamB-VD4-2, with a two amino acid overlap (Table 1). The N-terminal VD4-1 and C-terminal VD4-2 correspond to the postulated subspecies-specific and serovar-specific domains of VD4 in C. trachomatis respectively (Stephens et al., 1988). After religation of annealed pairs into the BamHI site of pAJC264 and transformation of E. coli $\mathrm{DH} 5 \alpha$, recombinant plasmids were characterized by restriction and nucleotide sequence analyses. Expressed LamB-VD1, -VD2, -VD3 and -VD4-2 hybrid polypeptides were of the expected sizes with the expected molecular mass increments of 1.5 to $2.5 \mathrm{kDa}$ and reacted strongly with polyclonal antisera against LamB and MOMP by immunoblot (Fig. 3). Hybrid polypeptide LamB-VD4-1 had a somewhat lower apparent molecular mass than expected (predicted $49.5 \mathrm{kDa}$ ). However, the presence of the appropriate VD4-1 insert in the LamB site was confirmed by nucleotide sequence analysis and by immunoblot.

A recombinant expressing a larger domain of GPIC MOMP was made by insertion of a BglII-HindIII fragment (nucleotides 486-1052 of the GPIC MOMP structural gene) into the BamHI-HindIII restricted expression vector pATH10 (Spindler et al., 1984). E. coli RR1 cells harbouring this plasmid construct (pGM101) express a $58 \mathrm{kDa}$ hybrid polypeptide (TrpE-MOMP) which includes the $37 \mathrm{kDa} \mathrm{N}$ terminal-most portion of the TrpE protein and a $21 \mathrm{kDa}$ MOMP segment at the C-terminal end (Table 1, Fig. 3).

Assessment of response to immunization. Serum and genital secretions were obtained by established methods (Rank et al., 1988) from each animal immediately prior to challenge infection, 2 weeks after the third immunization. Antibody titres in serum and genital secretions were determined by an enzyme immunoassay (EIA) using whole GPIC EBs (Rank et al., 1988). Antigen-specific antibody binding was assessed initially using immunoblot analyses using whole EBs as antigen (Batteiger \& Rank, 1987). GPIC EBs grown in McCoy cells were used as antigen in both EIA and immunoblot. Peripheral blood mononuclear cells were obtained prior to challenge infection, and blastogenic responses of such cells to whole GPIC EBs were determined according to established methods (Rank et al., 1988).

The humoral responses against linear epitopes contained in the specific VDs of GPIC MOMP were assessed by immunoblot using cell lysates from each of the recombinants described above. Radioiodinated staphylococcal protein A was used to detect bound antibody. Antigen preparations were adjusted to contain approximately $1 \mu \mathrm{g}$ of recombinant protein and sera were used at a single dilution $(1: 2000)$. Immunoblots were exposed for $24-48 \mathrm{~h}$ initially and then re-exposed for $10-14 \mathrm{~d}$ to confirm negative results.

\section{Results}

\section{Purification of GPIC proteins}

Fig. 1(a) shows a SDS- $12 \cdot 5 \%$ polyacrylamide gel containing resolved proteins of OGP-MOMP stained with Coomassie blue R-250. SDS-MOMP and SDS$61 \mathrm{kDa}$ were single entities when similarly analysed (not shown). The OGP-MOMP (Fig. 1 a) contained primarily MOMP, but other minor bands were visible, including bands in the region of the $84 \mathrm{kDa}$ outer membrane proteins, and $47 \mathrm{kDa}$ and $33 \mathrm{kDa}$ outer membrane proteins (Batteiger \& Rank, 1987). We estimated the purity of MOMP to be approximately $80 \%$ based on densitometric scanning of Coomassie blue-stained gels. Our results thus differ somewhat from those reported for C. trachomatis strain $\mathrm{L}_{2} / 434$ by Bavoil et al. (1984) who achieved $>90 \%$ purity assessed by a similar method. The more prominent contaminant bands observed here may have been due in part to differences in solubility of some outer membrane proteins in the $C$. psittaci strain as compared to the $C$. trachomatis strain.

\section{Immune response of immunized guinea-pigs}

Fig. $1(b, c)$ depicts immunoblots showing the qualitative responses of five animals to immunization with each of the two MOMP preparations from Experiment 2 (Table 2). Mock-immunized animals were negative by immunoblot (not shown).

Fig. 1(b) shows the serum IgG response by immunoblot of the five animals immunized with the SDS-MOMP 
(a)

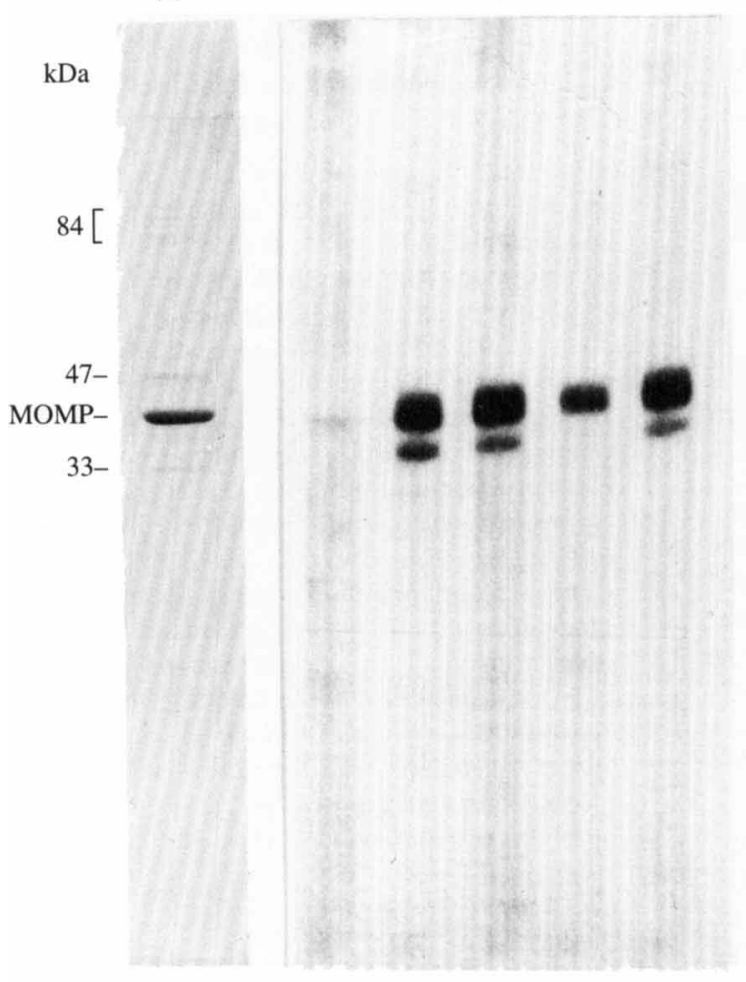

SDS-MOMP $(c)$

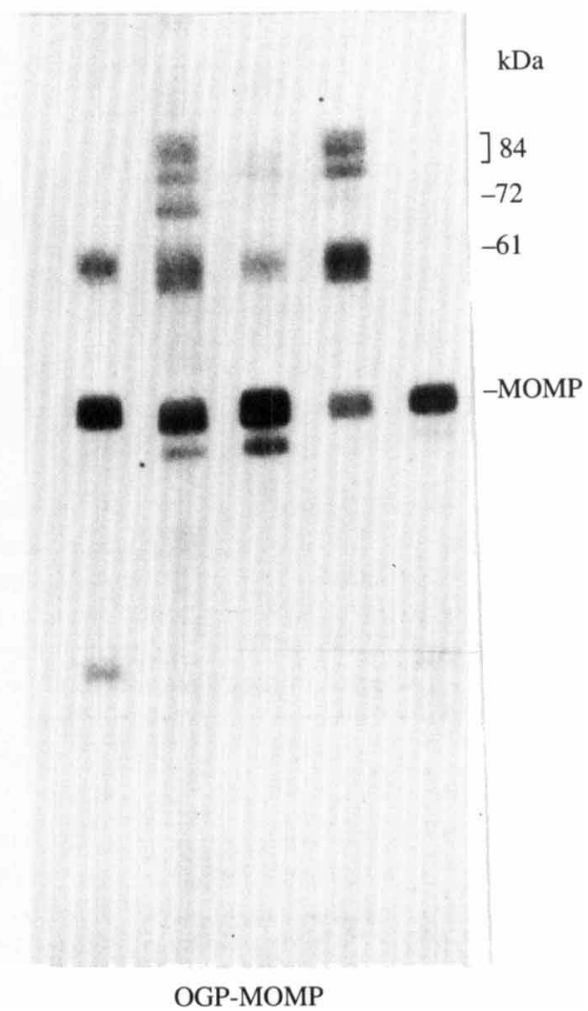

Fig. 1. (a) Coomassie blue-stained $12.5 \%$ polyacrylamide gel containing resolved proteins of chlamydial major outer membrane protein (MOMP) isolated using octyl- $\beta$-D-glucopyranoside (OGP) as described in the text. Identity of bands are noted on the left. $(b)$ Autoradiogram of the serum IgG responses of the five guinea-pigs from Experiment 2 which were immunized with MOMP isolated from SDS-PAGE gels. $(c)$ Autoradiogram of the serum IgG responses of the five animals from Experiment 2 which were immunized with OGP-MOMP. Identity of bands are marked on the right.

Table 2. Immune responses in immunized animals

Antibody titres are expressed as mean $\log _{10}$ (standard deviation).

\begin{tabular}{|c|c|c|c|c|c|c|}
\hline Group & Experiment & $\begin{array}{c}\text { Serum IgG } \\
\text { EIA }\end{array}$ & $\begin{array}{l}\text { Serum IgG } \\
\text { immunoblot }\end{array}$ & $\begin{array}{l}\text { Secretion } \\
\text { IgG EIA }\end{array}$ & $\begin{array}{l}\text { Secretion } \\
\text { IgA EIA }\end{array}$ & $\begin{array}{l}\text { Lymphocyte } \\
\text { proliferation } \dagger\end{array}$ \\
\hline \multirow[t]{2}{*}{ OGP-MOMP } & 1 & $3 \cdot 2 *(0 \cdot 25)$ & $4 \cdot 01$ & $2 \cdot 1 *(0 \cdot 25)$ & ND & $\begin{array}{c}24290 \\
(20381)\end{array}$ \\
\hline & 2 & $4 \cdot 01^{*}$ & $4 \cdot 01$ & $3 \cdot 6^{*}(0.27)$ & $3 \cdot 0 *(0 \cdot 16)$ & ND \\
\hline \multirow[t]{2}{*}{ SDS-MOMP } & 1 & $0.40(0.55)$ & $3.5(0.58)$ & 0 & $\mathrm{ND}$ & $\begin{array}{l}8738 \\
(2046)\end{array}$ \\
\hline & 2 & $1.8 \quad(0 \cdot 25)$ & $3.9(0.13)$ & $1 \cdot 2(0 \cdot 13)$ & $0.8 \quad(0.5)$ & ND \\
\hline SDS-61 & $\begin{array}{l}3 \\
4\end{array}$ & $\begin{array}{l}0.40(0.55) \\
1.2(0.16)\end{array}$ & $\begin{array}{l}\geqslant 2 \cdot 0 \\
\geqslant 2 \cdot 0\end{array}$ & $\begin{array}{l}\mathrm{ND} \\
\mathrm{ND}\end{array}$ & $\begin{array}{l}\text { ND } \\
\text { ND }\end{array}$ & $\begin{array}{c}\text { ND } \\
12946 \\
(10397)\end{array}$ \\
\hline Controls & $1-4$ & 0 & 0 & 0 & $\begin{array}{c}0 \\
(1374)\end{array}$ & 2440 \\
\hline
\end{tabular}

ND, Not Determined.

${ }^{*} P<0.001$ compared to SDS-MOMP group.

† Counts per minute (standard deviation).

preparation in Experiment 2. All animals responded by producing antibodies that bound MOMP; however, one animal responded only weakly. IgG in secretions (not shown) paralleled that of sera. A second band was sometimes seen at a position in the gel corresponding to about $35 \mathrm{kDa}$ (Fig. 1b). We do not know whether this 
Table 3. Reactivity of sera from immunized and infected guinea-pigs against recombinant MOMP fragments

Number of positive sera/number of animals tested in each group. Sera were called negative only when the level of reactivity remained undetectable above background after a prolonged exposure (see text).

\begin{tabular}{lccc}
\hline \hline & $\begin{array}{c}\text { Guinea-pigs } \\
\text { immunized with } \\
\text { SDS-MOMP }\end{array}$ & $\begin{array}{c}\text { Guinea-pigs } \\
\text { immunized with } \\
\text { OGP-MOMP }\end{array}$ & $\begin{array}{c}\text { Guinea-pigs } \\
\text { infected in the } \\
\text { genital tract }\end{array}$ \\
\hline TrpE & $0 / 5$ & $0 / 4$ & $0 / 5$ \\
TrpE-MOMP & $5 / 5$ & $4 / 4$ & $25 / 29$ \\
LamB & $0 / 9$ & $1 / 4$ & $0 / 29$ \\
LamB-VD1 & $8 / 9$ & $4 / 4$ & $26 / 29$ \\
LamB-VD2 & $3 / 9$ & $3 / 4$ & $24 / 29$ \\
LamB-VD3 & $6 / 9$ & $3 / 4$ & $2 / 4$ \\
LamB-VD4-1 & $6 / 9$ & $4 / 4$ & $3 / 4$ \\
LamB-VD4-2 & $6 / 9$ & $3 / 4$ & $6 / 29$ \\
\hline
\end{tabular}

band represented antibodies formed in response to a protein contaminating the immunogen, or MOMP antibodies that bound an altered (e.g. proteolytically degraded or internally disulphide-linked) form of MOMP.

Fig. 1(c) shows the serum IgG responses by immunoblot of five animals immunized with OGP-MOMP in Experiment 2. All sera contained antibodies to MOMP and also to the $35 \mathrm{kDa}$ band described above. In addition, $4 / 5(9 / 10$ overall) gave responses to the $61 \mathrm{kDa}$ outer membrane protein, and $3 / 5(8 / 10$ overall) to the ca. $84 \mathrm{kDa}$ outer membrane proteins. These results indicate that the OGP-MOMP preparation was contaminated with enough $61 \mathrm{kDa}$ to elicit a detectable IgG response, but not to give a visible band in Coomassiestained gels. Although the $47 \mathrm{kDa}$ and $33 \mathrm{kDa}$ proteins were visible contaminants by SDS-PAGE analysis, they did not elicit an antibody response detectable by immunoblot. In Experiment $1,5 / 5$ animals in the OGPMOMP group had binding at the region of LPS, suggesting that LPS was present in the first lot of immunogen. However, in Experiment 2 (Fig. 1c), no sera bound antibody at the region of LPS.

Quantitative humoral immune responses for all animals both by EIA and immunoblot are given in Table 2. When assessed by immunoblot, there was no statistical difference in the uniformly high antibody titres against MOMP between animals immunized with OGP-MOMP and SDS-MOMP (Table 2). In contrast, when assessed by whole EB EIA, the OGP-MOMP preparation elicited a significantly higher antibody response than the SDSMOMP preparation, both in serum and secretions $(P<0.001$, Table 2$)$. Antibody titres measured by EIA and immunoblot were not statistically different in the OGP-MOMP group, whereas in the SDS-MOMP group,

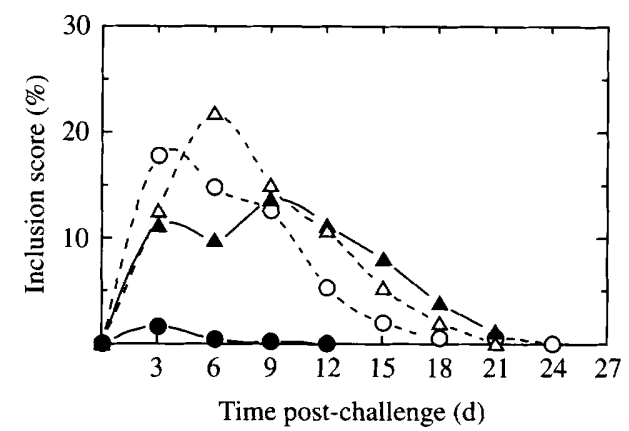

Fig. 2. Course of genital infection of groups (10 animals in each group) immunized with OGP-MOMP (O), OGP-control (O), SDS-MOMP $(\Delta)$, and SDS-control $(\triangle)$. OGP- and SDS-controls were mock immunized with detergent in buffer only. Inclusion scores were determined as described in the text and are expressed as a percentage of epithelial cells examined. Lack of a symbol at any given day indicates that inclusions were not found in the genital smears from that day.

EIA titres were significantly lower than immunoblot titres $(P<0.001)$.

While the blastogenic responses were higher in the OGP-MOMP group than in the SDS-MOMP group, the difference in the mean response was not statistically significant (Table 2).

Animals immunized with SDS-61 kDa had serum IgG responses against the $61 \mathrm{kDa}$ protein at titres $\geqslant 1: 100$ in immunoblot. Titres in EIA are shown in Table 2.

\section{Response to challenge infection}

The summed courses of infections for the 10 animals receiving each treatment is shown in Fig. 2. The course of infection in groups of animals immunized with either SDS-MOMP or SDS-61 kDa did not differ from the infections observed in the control groups. Thus, no protection was observed despite the immune responses described above. In contrast, the group of 10 animals immunized with OGP-MOMP had significantly reduced intensity and duration of infection as measured by inclusion scores $(P<0.0001)$, although duration of chlamydial shedding detected by cell culture did not differ from the control group. We saw no difference in the courses of infection in the OGP-MOMP groups in Experiment 1 (with LPS antibodies) versus Experiment 2 (no LPS antibodies).

\section{Fine specificity of serum antibodies to MOMP}

Recombinant $E$. coli cells expressing LamB-VD1, -VD2, -VD3 and LamB fused to VD4-1 (N-terminal half) and VD4-2 (C-terminal half) served as antigen in immunoblots to analyse the specificity of serum antibodies from guinea pigs immunized with either OGP-MOMP or SDS-MOMP. In addition, sera from 29 animals which 
(a)
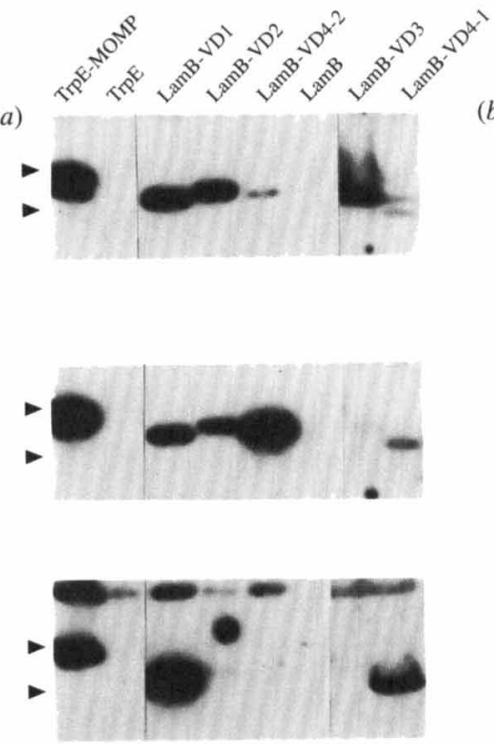

Genital infection (b)
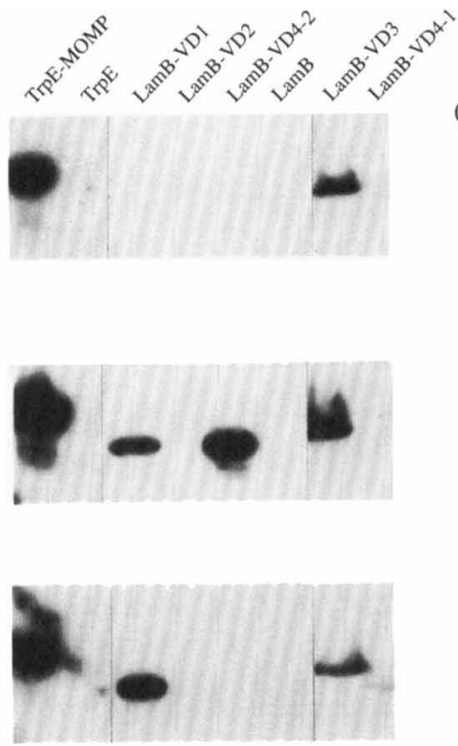

SDS-MOMP $(c)$
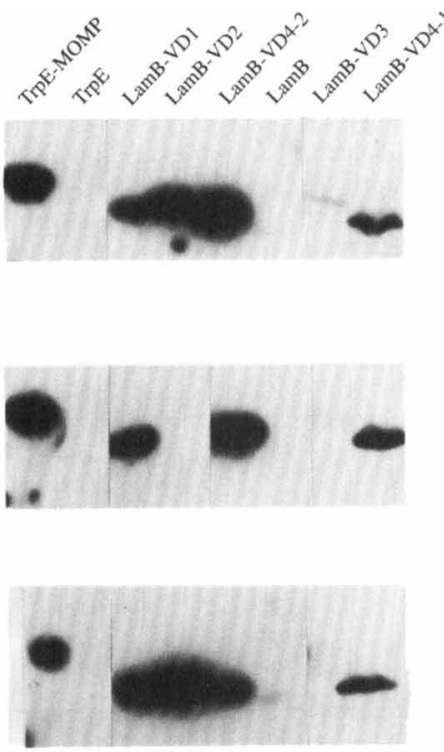

OGP-MOMP

Fig. 3. Autoradiograms of IgG reactions in immunoblots of representative sera to recombinant peptides representing the variable domains of the MOMP of the guinea-pig inclusion conjunctivitis agent. Panels $a, b$ and $c$ represent the results of a serum from each of three animals used to probe a panel of eight recombinant antigens from $E$. coli lysates. (a) Sera from three animals which recovered from a genital infection and were thus partially protected against challenge infection. (b) Sera from three animals immunized with SDSMOMP and were thus unprotected against challenge infection. (c) Sera from three animals immunized with OGP-MOMP and were thus partially protected against challenge infection. Arrowheads on the left indicate the positions of the fusion proteins containing chlamydial antigens.

had recovered from a genital infection and were resistant to re-challenge (Rank et al., 1988) were included for comparison.

Sera from all immunized animals gave strong reactions with TrpE-MOMP (Table 3), confirming results obtained with whole MOMP (Fig. 1), while 25/29 infected animals gave reactions to TrpE-MOMP (Table 3). Reactions of sera with the various recombinant VDs were not uniform (Table 3) and included sera from each group which did not react with one or more of the recombinant peptides (Fig. 3). Table 3 shows that animals immunized with SDS-MOMP reacted less frequently to LamB-VD2 as compared to animals in the OGP-MOMP-immunized group, while the group of animals which had recovered from genital infection reacted less frequently to LamBVD4-2. However, the analysis failed to reveal statistically significant differences in reactivity among the three groups of sera for any recombinant peptide.

\section{Discussion}

Our results indicate that a protective immune response can be elicited in the guinea-pig: GPIC model of genital chlamydial infection using a refined vaccine (OGPMOMP) derived from outer membrane complexes. The vaccine was largely MOMP, but in most animals elicited antibodies observed by immunoblot that bound other outer membrane components, including the $61 \mathrm{kDa}$, $84 \mathrm{kDa}$ and $72 \mathrm{kDa}$ outer membrane proteins. Genital infection with the GPIC agent frequently induces antibodies to these outer membrane proteins (Batteiger $\&$ Rank, 1987). Thus, we cannot conclusively determine whether the observed protection was the result of immune responses elicited by MOMP itself, or the result of responses elicited by contaminant outer membrane proteins. However, it should be noted that one animal in the OGP-MOMP group had a monospecific response to MOMP and showed the same degree of protection as the other animals. In either case, we have shown that a parenterally administered subunit vaccine can induce detectable protection against mucosal infection as defined by a marked decrease in intensity of infection. Histopathology of the lower and upper genital tract was not evaluated as a part of this study.

Our results also indicate that SDS-MOMP, as prepared and administered in this study, was capable of inducing antibodies to MOMP in both serum and secretions, but incapable of inducing protection. Likewise, a vaccine prepared from SDS-purified $61 \mathrm{kDa}$ outer membrane protein induced measurable immune responses, but afforded no protection.

The SDS-MOMP vaccine elicited strong antibody 
responses that could be measured by immunoblot, but only weak antibody responses measured by EIA. Blastogenic responses were also weak. The EIA assay, using whole EBs as antigen, detects primarily antibodies to surface-exposed antigens. It is possible that the SDSMOMP vaccine elicited responses to cross-reactive but surface inaccessible antigens which are not involved in eliciting a protective response. The same patterns held for the SDS-61 kDa immunogen. The analogue of the latter protein has been shown not to be surface exposed in C. trachomatis strains (Collett et al., 1989), even though it frequently induces a prominent humoral response in genital infection in humans (Newhall et al., 1982) and guinea-pigs (Batteiger \& Rank, 1987).

In contrast, the OGP-MOMP elicited strong antibody responses both in immunoblot and in EIA, and elicited marginally more vigorous blastogenic responses. As compared to SDS-MOMP, it is possible that MOMP in the OGP preparation has a conformation more closely approximating that of its native configuration and thus either elicited a protective response to discontinuous epitopes or a more efficient response to protective linear epitopes.

In an attempt to identify regions of MOMP differentially recognized by anti-MOMP antibodies from unprotected (SDS-MOMP-immunized) and protected (post-infection and OGP-MOMP-immunized) animals, recombinants expressing peptides corresponding to the immunodominant VDs of MOMP were used to analyse the sera. While virtually all antisera from all three groups contained high titres of antibodies to whole and recombinant MOMP by immunoblot, responses to individual VDs were more variable. Thus, there were no VD regions to which all protected animals responded but to which no unprotected animals responded. Although the proportions of animals with reactive sera varied by group, no statistically significant differences and thus no striking correlations of reactivity and protection were found.

The observed protection was only partial, in that organisms were shed, albeit at low levels, even in the OGP-MOMP animals. The reason for this is not clear but could be related to an inability of the subcutaneous vaccine to elicit an adequate cell-mediated response. This could result either from a failure to elicit appropriate effector cells or failure to elicit those $T$ cells which are able to home to the mucosa-associated lymphoid tissue in the genital tract. We have previously demonstrated that cell-mediated immunity is essential for the resolution of a chlamydial genital infection (Rank et al., 1989). Absolute prevention of infection may be difficult to achieve, since even in the natural infection, such immunity is short-lived (Rank et al., 1988). A more appropriate goal might be the reduction of the level of infection and prevention of ascending infection resulting in salpingitis. Thus, studies are in progress to determine whether subunit immunizations influence the occurrence of such complications in female guinea-pigs.

This study was supported by Public Health Service grants AI-23044 (R.G. R., B.E. B.) and AI-26280 (P. M. B.) from the National Institutes of Health, and a grant from the Edna McConnell Clark Foundation (P.M. B.). We thank Dr W. J. Newhall for useful discussions and Mary Stenstrom, Teresa Lewis, Lynn McAlister, Eric Ingerowski and Lisa Loeffelholz for excellent technical assistance.

\section{References}

Bafhr, W., Zhang, Y.-X., Joseph, T., Su, H., Nano, F. E., Everett, K. D. E. \& CALDWELL, H. D. (1988). Mapping antigenic domains expressed by Chlamydia trachomatis major outer membrane protein. Proceedings of the National Academy of Sciences of the United States of America 85, 4000-4004.

Batteiger, B. E. \& Rank, R. G. (1987). Analysis of the humoral immune response in chlamydial genital infection in guinea pigs. Infection and Immunity 55, 1767-1773.

Batteiger, B. E., Newhall, W. J. V. \& Jones, R. B. (1985). Differences in outer membrane proteins of the lymphogranuloma venereum and trachoma biovars of Chlamydia trachomatis. Infection and Immunity 50, 488-494.

Bavorl, P., Ohlin, A. \& Schachter, J. (1984). Role of disulfide bonding in outer membrane structure and permeability in Chlamydia trachomatis. Infection and Immunity 44, 479-485.

BRADFORD, M. M. (1976). A rapid and sensitive method for the quantitation of microgram quantities of protein utilizing the principle of protein-dye binding. Analytical Biochemistry 72, 248-254.

Caldwell, H. D. \& SChachter, J. S. (1982). Antigenic analysis of the major outer membrane protein of Chlamydia spp. Infection and Immunity 35, 1024-1031.

Caldwell, H. D., Kromhout, J. \& Schachter, J. S. (1981). Purification and partial characterization of the major outer membrane protein of Chlamydia trachomatis. Infection and Immunity 31, $1161-1176$.

Charbit, A., Boulain, J.-C., Ryter, A. \& Hofnung, M. (1986). Probing the topology of a bacterial membrane protein by genetic insertion of a foreign epitope: expression at the cell surface. $E M B O$ Journal 5, 3029-3037.

Collett, B. A., Newhall, W. J. V., Jersild, R. A., JR \& Jones, R. B. (1989). Detection of surface-exposed epitopes on Chlamydia trachomatis by immune electron microscopy. Journal of General Microbiology 135, 85-94.

LAEMmLI, U. K. (1970). Cleavage of structural proteins during the assembly of the head of bacteriophage T4. Nature, London 227 , 680-685.

Morrison, R. P., Lyng, K. \& Caldwell, H. D. (1989). Chlamydial disease pathogenesis: ocular hypersensitivity elicited by a genusspecific 57-kD protein. Journal of Experimental Medicine 169 , 663-675.

Newhall, W. J. V., Batteiger, B. E. \& Jones, R. B. (1982). Analysis of the human serological response to proteins of Chlamydia trachomatis. Infection and Immunity 38, 1181-1189.

RANK, R. G. \& BARRON, A. L. (1987). Specific effect of estradiol on the genital mucosal antibody response in chlamydial ocular and genital infections. Infection and Immunity 55, 2317-2319.

Rank, R. G. \& Batteiger, B. E. (1989). Protective role of serum antibody in immunity to chlamydial genital infection. Infection and Immunity 57, 299-301.

Rank, R. G., Batteiger, B. E. \& Soderberg, L. S. F. (1988). Susceptibility to reinfection after a primary chlamydial genital infection. Infection and Immunity 56, 2243-2249.

Rank, R. G., Soderberg, L. S. F., Sanders, M. M. \& Batteiger, B. E. (1989). Role of cell-mediated immunity in the resolution of secondary chlamydial genital infection in guinea pigs infected with 
the agent of guinea pig inclusion conjunctivitis. Infection and Immunity 57, 706-710.

SCHACHTER, J. (1985). Overview of Chlamydia trachomatis infection and the requirements for a vaccine. Reviews of Infectious Diseases 7 , 713-716.

SCHACHTER, J. \& Dawson, C. R. (1978). Human Chlamydial Infections. Littleton, Mass: PSG Publishing Co.

Spindler, K. R., Rosser, D. S. E. \& Berk, A. J. (1984). Analysis of adenovirus transforming proteins from early regions $1 \mathrm{~A}$ and $1 \mathrm{~B}$ with antisera to inducible fusion antigens produced in Escherichia coli. Journal of Virology 49: 132-141.

Stephens, R. S., Wagar, E. A. \& Schoolnik, G. K. (1988). Highresolution mapping of serovar-specific and common antigenic determinants of the major outer membrane protein of Chlamydia trachomatis. Journal of Experimental Medicine 167, 817-831.

Su, H., Zhang, Y.-X., Barrera, O., Watkins, N. \& Caldwell, H. D. (1988). Differential effect of trypsin on infectivity of Chlamydia trachomatis: loss of infectivity requires cleavage of major outer membrane protein variable domains II and IV. Infection and Immunity 56, 2094-2100.
Tan, T.-W., Herring, A. J., Anderson, I. E. \& Jones, G. E. (1990). Protection of sheep against Chlamydia psittaci infection with a subcellular vaccine containing the major outer membrane protein. Infection and Immunity 58, 3101-3108.

Taylor, H. R., Whittum-Hudson, J., Schachter, J., Caldwell, H. D. \& PRENDERGast R. A. (1988). Oral immunization with chlamydial major outer membrane protein (MOMP). Investigative Ophthalmology and Visual Science 29, 1847-1853.

Yuan, Y., Zhang, Y.-X., Watkins, N. G. \& Caldwell, H. D. (1989). Nucleotide and deduced amino acid sequences for the four variable domains of the major outer membrane proteins of the 15 Chlamydia trachomatis serovars. Infection and Immunity 57, 1040-1049.

Zhang, Y.-X., Stewart, S., Joseph, T., Taylor, H. R. \& Caldwell, H. D. (1987). Protective monoclonal antibodies recognize epitopes located on the major outer membrane protein of Chlamydia trachomatis. Journal of Immunology 138, 575-581.

Zhang, Y.-X., Morrison, S. G., Caldwell, H. D. \& Baehr, W. (1989). Cloning and sequence analysis of the major outer membrane protein genes of two Chlamydia psittaci strains. Infection and Immunity 57, 1621-1625. 